\section{\$41. Reduction of Sheath Potential and Particle Flux at a Target Plate by Negatively Charged Dust Particles}

Tomita, Y., Takayama, A., Smimov, R. (Grad. Univ. Adv. Studies), Chutov, Yu. (Kiev Univ.), Takizuka, T. (Naka Fusion Res. Establishment, JAERI)

Electrostatic potential formation due to negatively charged dust particles near a divertor plate is studied by one-dimensional kinetic analysis. It is shown that the negatively charged dust particles reduce the ionsheath potential drop in front of the plate. Dust particles with a large radius $(100 \mu \mathrm{m})$ reduce the potential drop significantly despite the low dust-to-plasma density ratio $\left(\sim 10^{-8}\right)$. The plasma particle flux is reduced appreciably by the absorption by the dust particles: 1 )

In our one-dimensional model geometry, plasma particles with half-Maxwellian distribution functions are injected from an injection position $(x=0)$ along the magnetic field toward a perfectly absorbing divertor or target plate at floating potential $\left(x=x_{\mathrm{w}}\right)$, with immovable dust particles distributed uniformly. In this analysis we investigate the formation of a potential decreasing monotonically toward the divertor platc, with the potential at the injection point being zero. The absorption of plasma particles by dust particles and Coulomb interactions between particles are neglected because the mean free paths are much longer than the system length, which is assumed to be of the order of the Debye length. The negatively charged dust particles affect the potential formation through Poisson's equation. In the model system without plasma sources and sinks, the local energy distribution function is described by the injection distribution. These local energy distribution functions yield the local macroscopic quantities. The local charge density of the dust particles is calculated according to the absorption cross-section from OML theory 2).

The spatial potential profiles for several values of the normalized dust density $n_{d}^{*} R_{d}^{*}\left(=n_{d} R_{d} \lambda_{D e s}^{2}\right)$ are given in Fig.1, where the floating target is located at the position $x_{w}=50 \lambda_{\text {Des }}$ and $\lambda_{\text {Des }}$ is the Debye length at the injection point. Near the injection point an electron sheath is formed because the electron density is higher than the ion density, and in the middle region this system exhibits a wide quasi-neutral region. In front of the floating target plate an ion-sheath is formed. Note that the wall potential is not affected by the immovable dust particles. The normalized potential drop between the charge-neutrality point and the wall is shown in Fig. 2 as a function of the normalized dust density.

For a dust-particle radius of $1 \mu \mathrm{m}$, dust-to-plasma density ratios of the order of $10^{-6}$ effectively reduce the sheath-potential drop in divertor plasmas of fusion devices. Large dust-particle radii $(\sim 100 \mu \mathrm{m})$ reduce the sheath potential drop more effectively, even at low dust-to-plasma density ratios $\left(\sim 10^{-8}\right)$. In this case the reduction of the plasma flux to the divertor plate is appreciable due to the absorption of plasma particles by the dust particles. In this simplified analytical study, several issues have been left out which are required for understanding dust-particle effects in a realistic divertor plasma: The presheath, which can be governed by geometric, collisional, ionization, or magnetic-field effects, is important for determining the spatial distribution of dust particles. Moreover, the magnetic field obliquely penetrating the divertor plate affects the potential formation in the presence of dust particles. Finally, from our results the effect of plasmaparticle absorption by dust particles was found to be nonnegligible, which means that this effect needs to be incorporated in the model as well.

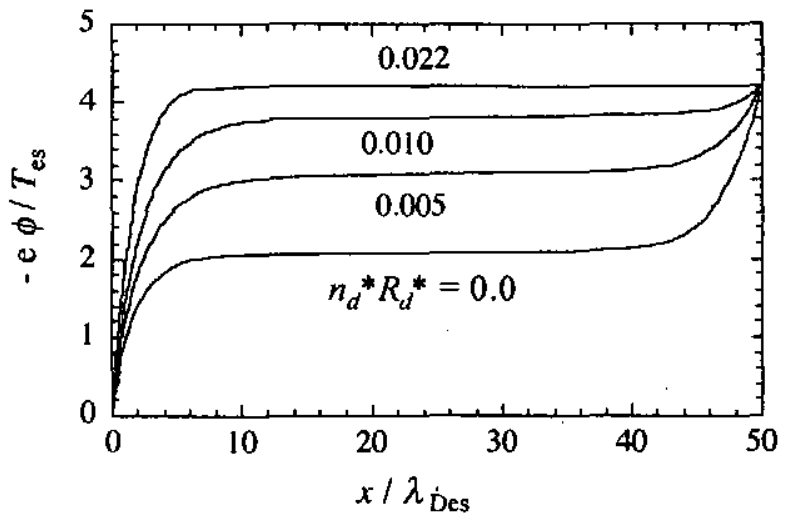

Fig.1. Spatial distributions of the normalized electrostatic potential $-e \phi / T_{e s}$ for several values of the dust density. $T_{e s}$ is the electron temperature at the injection point.

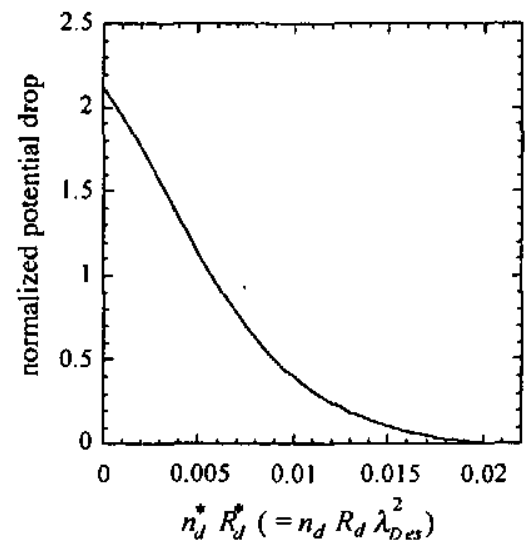

Fig.2. Normalized potential drop between the chargeneutrality point and the wall as a function of the normalized dust density.

\section{References}

1) Y. Tomita , R. Smirnov, Yu. Chutov, A. Takayama, and T. Takizuka, Contrib. Plasma Phys., 44 (2004) 162.

2) J. E. Allen, Physica Scripta, 45 (1992) 497. 